\title{
UNIQUENESS OF POSITIVE RADIAL SOLUTIONS TO SINGULAR CRITICAL GROWTH QUASILINEAR ELLIPTIC EQUATIONS
}

\section{Cheng-Jun He and Chang-Lin Xiang}

Chinese Academy of Sciences, Wuhan Institute of Physics and Mathematics

P. O. Box 71010, Wuhan, 430071, P. R. China; cjhe@wipm.ac.cn

University of Jyväskylä, Department of Mathematics and Statistics

P. O. Box 35, FI-40014 University of Jyväskylä, Finland; Xiang_math@126.com

Abstract. In this paper, we prove that there exists at most one positive radial weak solution to the following quasilinear elliptic equation with singular critical growth

$$
\begin{cases}-\Delta_{p} u-\frac{\mu}{|x|^{p}}|u|^{p-2} u=\frac{|u|^{\frac{(N-s) p}{N-p}-2} u}{|x|^{s}}+\lambda|u|^{p-2} u & \text { in } B \\ u=0 & \text { on } \partial B\end{cases}
$$

where $B$ is an open finite ball in $\mathbf{R}^{N}$ centered at the origin, $1<p<N,-\infty<\mu<((N-p) / p)^{p}$, $0 \leq s<p$ and $\lambda \in \mathbf{R}$. A related limiting problem is also considered.

\section{Introduction and main results}

In this paper, we consider the following quasilinear elliptic equation

$$
\begin{cases}-\Delta_{p} u-\frac{\mu}{|x|^{p}}|u|^{p-2} u=\frac{|u|^{p^{*}(s)-2} u}{|x|^{s}}+\lambda|u|^{p-2} u & \text { in } B, \\ u=0 & \text { on } \partial B,\end{cases}
$$

where $B$ is an open finite ball in $\mathbf{R}^{N}$ centered at the origin, $1<p<N,-\infty<\mu<$ $\bar{\mu}=((N-p) / p)^{p}, 0 \leq s<p, p^{*}(s)=(N-s) p /(N-p), \lambda \in \mathbf{R}$ and

$$
\Delta_{p} u=\sum_{i=1}^{N} \partial_{x_{i}}\left(|\nabla u|^{p-2} \partial_{x_{i}} u\right), \quad \nabla u=\left(\partial_{x_{1}} u, \cdots, \partial_{x_{N}} u\right)
$$

is the $p$-Laplacian operator.

It is well known that equation (1.1) is the Euler-Lagrange equation of the energy functional $J: W_{0}^{1, p}(B) \rightarrow \mathbf{R}$ defined as

$$
J(u)=\frac{1}{p} \int_{B}\left(|\nabla u|^{p}-\frac{\mu}{|x|^{p}}|u|^{p}-\lambda|u|^{p}\right) d x-\frac{1}{p^{*}(s)} \int_{B} \frac{|u|^{p^{*}(s)}}{|x|^{s}} d x, \quad u \in W_{0}^{1, p}(B),
$$

where the Sobolev space $W_{0}^{1, p}(B)$ is the completion of $C_{0}^{\infty}(B)$, the space of smooth functions with compact support in $B$, in the seminorm $\|u\|_{W_{0}^{1, p}(B)}=\|\nabla u\|_{L^{p}(B)}$. All the integrals in functional $J$ are well defined, due to the Hardy inequality $[13,19]$

$$
\left(\frac{N-p}{p}\right)^{p} \int_{B} \frac{|\varphi|^{p}}{|x|^{p}} d x \leq \int_{B}|\nabla \varphi|^{p} d x, \quad \forall \varphi \in W_{0}^{1, p}(B),
$$

doi:10.5186/aasfm.2016.4110

2010 Mathematics Subject Classification: Primary 35A24, 35B33, 35B40, 35J75, 35J92.

Key words: Quasilinear elliptic equations, singular critical growth, positive radial solutions, Pohozaev identity, uniqueness, asymptotic behaviors.

This is part of the $\mathrm{PhD}$ work of the second named author at the University of Jyväskylä. 
and due to the Caffarelli-Kohn-Nirenberg inequality [6]

$$
C\left(\int_{B} \frac{|\varphi|^{p^{*}(s)}}{|x|^{s}} d x\right)^{\frac{p}{p^{*}(s)}} \leq \int_{B}|\nabla \varphi|^{p} d x, \quad \forall \varphi \in W_{0}^{1, p}(B),
$$

where $C=C(N, p, s)>0$.

We say that a function $u \in W_{0}^{1, p}(B)$ is a weak solution to equation (1.1), if for all functions $\varphi \in C_{0}^{\infty}(B)$, we have

$$
\int_{B}\left(|\nabla u|^{p-2} \nabla u \cdot \nabla \varphi-\frac{\mu}{|x|^{p}}|u|^{p-2} u \varphi-\lambda|u|^{p-2} u \varphi\right) d x=\int_{B} \frac{|u|^{p^{*}(s)-2} u}{|x|^{s}} \varphi d x .
$$

In the following, we will systematically omit the word "weak" and simply say that $u$ is a solution to equation (1.1), meaning (1.2); a similar convention for weak solutions to equations in the below.

By Theorem 1.1 of Han [18], we have the following existence result: Consider equation (1.1) with $s=0$, that is,

$$
\begin{cases}-\Delta_{p} u-\frac{\mu}{|x|^{p}}|u|^{p-2} u=|u|^{p^{*}-2} u+\lambda|u|^{p-2} u & \text { in } B, \\ u=0 & \text { on } \partial B\end{cases}
$$

where we write $p^{*}=p^{*}(0)$ for simplicity throughout the paper. Assume that $1<$ $p^{2}<N$ and $0<\mu \leq N^{p-1}\left(N-p^{2}\right) / p^{p}$. Then for every $\lambda, 0<\lambda<\lambda_{1}(\mu)$, there exists at least one positive solution to equation (1.3), where $\lambda_{1}(\mu)$ is defined by

$$
\lambda_{1}(\mu)=\inf _{u \in W_{0}^{1, p}(B) \backslash\{0\}} \frac{\int_{B}\left(|\nabla u|^{p}-\mu|x|^{-p}|u|^{p}\right) d x}{\int_{B}|u|^{p} d x} .
$$

In the case $p=2$, above existence result was also obtained by Jannelli [21] on more general domains. For more results on existence of solutions to equation (1.1) and its variants, we refer to e.g. [4, 7, 8, 9, 16, 17, 18, 21].

A very important ingredient in the argument of Han [18] is the following result, which was obtained by Abdellaoui, Felli and Peral [3]: Denote by $\mathcal{D}^{1, p}\left(\mathbf{R}^{N}\right)$ the completion of $C_{0}^{\infty}\left(\mathbf{R}^{N}\right)$, the space of smooth functions in $\mathbf{R}^{N}$ with compact support, in the seminorm $\|v\|_{\mathcal{D}^{1, p}\left(\mathbf{R}^{N}\right)}=\|\nabla v\|_{L^{p}\left(\mathbf{R}^{N}\right)}$. Consider the limiting problem

$$
-\Delta_{p} u-\frac{\mu}{|x|^{p}}|u|^{p-2} u=|u|^{p^{*}-2} u \quad \text { in } \mathbf{R}^{N},
$$

where $0<\mu<\bar{\mu}$. There is a unique ground state $U \in \mathcal{D}^{1, p}\left(\mathbf{R}^{N}\right)$ to equation (1.5), up to a dilation $U^{\tau}=\tau^{-(N-p) / p} U(\cdot / \tau), \tau>0$. Moreover, $U$ is a positive radial function which satisfies

$$
\lim _{|x| \rightarrow 0} U(x)|x|^{\gamma_{1}}=C_{1} \quad \text { and } \quad \lim _{|x| \rightarrow \infty} U(x)|x|^{\gamma_{2}}=C_{2}
$$

and

$$
\lim _{|x| \rightarrow 0}|\nabla U(x)||x|^{\gamma_{1}+1}=\left|\gamma_{1}\right| C_{1} \quad \text { and } \quad \lim _{|x| \rightarrow \infty}|\nabla U(x)||x|^{\gamma_{2}+1}=\gamma_{2} C_{2},
$$

where $C_{1}, C_{2}>0$ are constants depending only $N, p$ and $\mu$.

In the estimates (1.6) and (1.7), the exponents $\gamma_{1}, \gamma_{2}$ are defined as follows: Define $\Gamma_{\mu}: \mathbf{R} \rightarrow \mathbf{R}$ by

$$
\Gamma_{\mu}(\gamma)=(p-1)|\gamma|^{p}-(N-p)|\gamma|^{p-2} \gamma+\mu, \quad \gamma \in \mathbf{R} .
$$


Consider the equation

$$
\Gamma_{\mu}(\gamma)=0, \quad \gamma \in \mathbf{R}
$$

Due to our assumptions on $N, p$ and $\mu$, that is, $1<p<N$ and $-\infty<\mu<\bar{\mu}=$ $((N-p) / p)^{p}$, equation (1.9) admits two and only two solutions, denoted by $\gamma_{1}$ and $\gamma_{2}$, with $\gamma_{1}<\gamma_{2}$.

For later use, we note that in the case $0<\mu<\bar{\mu}$, we have

$$
0<\gamma_{1}<\frac{N-p}{p}<\gamma_{2}<\frac{N-p}{p-1}
$$

and in the case $\mu<0$, we have

$$
\gamma_{1}<0<\frac{N-p}{p-1}<\gamma_{2}
$$

In the case $\mu=0$, we have

$$
\gamma_{1}=0 \quad \text { and } \quad \gamma_{2}=\frac{N-p}{p-1}
$$

and in the case $p=2$, we have

$$
\gamma_{1}=\sqrt{\bar{\mu}}-\sqrt{\bar{\mu}-\mu} \text { and } \gamma_{2}=\sqrt{\bar{\mu}}+\sqrt{\bar{\mu}-\mu} .
$$

A natural question is whether the positive solution obtained by Han [18] to equation (1.3) is unique. In the case when $p=2$, the answer is affirmative, see Ramaswamy and Santra [30], where a more general uniqueness result was obtained. In the general case when $1<p<N$, this question has not yet been fully understood. In this paper, we give a partial answer to this question. In fact, we will prove a slightly more general result (see Theorem 1.2 below). As a consequence, we have the following uniqueness result.

Theorem 1.1. Assume that $1<p^{2}<N, 0<\mu \leq N^{p-1}\left(N-p^{2}\right) / p^{p}$ and $0<\lambda<\lambda_{1}(\mu)$. Then equation (1.3) admits at most one positive radial solution in $B$.

We remark that in the case $p=2$, positive solutions to equation (1.3) with $0<\mu<\bar{\mu}$ and $0<\lambda$ are radial by Lemma 3.1 of Ramaswamy and Santra [30], while in the general case $1<p<N, p \neq 2$, the symmetry of positive solutions to equation (1.3) with $0<\mu<\bar{\mu}$ and $0<\lambda$ seems to be unknown.

Note that the result of Theorem 1.1 dose not cover the full range of the parameters $p, \mu$ and $\lambda$. In this paper, we will prove the uniqueness of positive radial weak solutions to equation (1.1) in the full range of parameters of $p, \mu, s$ and $\lambda$, that is, $1<p<N$, $-\infty<\mu<\bar{\mu}=((N-p) / p)^{p}, 0 \leq s<p$ and $\lambda \in \mathbf{R}$. Our motivation to consider the full range of these parameters comes from the fact that, there have been extensive studies on problems of type (1.1) but with different ranges of these parameters and different ideas. For instance, to prove Theorem 1.2 below, nondegeneracy arguments were applied by Zhang [34], Srikanth [31] and Ramaswamy and Santra [30] when $p=2$, while a significantly different idea from those of $[34,31,30]$ was introduced by Adimurthi and Yadava [1] to deal with $p \neq 2$ and $\mu=s=0$. Therefore, it is preferable to derive a unified theory that covers the full range of those parameters. Furthermore, as a byproduct, deeper insights into the common features of equations of type (1.1) will be gained after such an investigation. 
We also consider the following limiting problem

$$
-\Delta_{p} u-\frac{\mu}{|x|^{p}}|u|^{p-2} u=\frac{|u|^{p^{*}(s)-2} u}{|x|^{s}} \quad \text { in } \mathbf{R}^{N} .
$$

It is easy to see that equation (1.5) is a special case of equation (1.10). A function $u \in \mathcal{D}^{1, p}\left(\mathbf{R}^{N}\right)$ is a (weak) solution to equation (1.10), if for every $\varphi \in C_{0}^{\infty}\left(\mathbf{R}^{N}\right)$, we have

$$
\int_{\mathbf{R}^{N}}\left(|\nabla u|^{p-2} \nabla u \cdot \nabla \varphi-\frac{\mu}{|x|^{p}}|u|^{p-2} u \varphi\right) d x=\int_{\mathbf{R}^{N}} \frac{|u|^{p^{*}(s)-2} u}{|x|^{s}} \varphi d x .
$$

We will only consider positive radial weak solutions to equation (1.10). In the following we discuss positive radial weak solutions to equation (1.1) and equation (1.10) respectively.

1.1. Uniqueness of positive radial weak solutions to equation (1.1). In this subsection, we consider equation (1.1). We are concerned with the uniqueness of positive radial solutions to equation (1.1). Uniqueness problems have been considered extensively in the literature. We refer the reader to e.g. [1, 12, 23, 30, 31, 34], where more general nonlinear elliptic equations were studied.

When $p=2$, equation (1.1) is reduced to

$$
\begin{cases}-\Delta u-\frac{\mu}{|x|^{2}} u=\frac{|u|^{2^{*}(s)-2} u}{|x|^{s}}+\lambda u & \text { in } B, \\ u=0 & \text { on } \partial B .\end{cases}
$$

When $\lambda \leq 0$, it is standard to prove that equation (1.11) admits no positive solution by Pohozaev identity [28]. When $0 \leq \mu<\bar{\mu}=(N-2)^{2} / 4, s=0$ and $\lambda>0$, it is well known $[14,30]$ that positive solutions to equation (1.11) are radial. When $\mu=s=0$ and $\lambda>0$, the uniqueness of positive solutions to equation (1.11) was proved by Zhang [34] and Srikanth [31], while for $0<\mu<\bar{\mu}=(N-2)^{2} / 4, s=0$ and $\lambda>0$, the uniqueness for positive solutions to equation (1.11) was proved by Ramaswamy and Santra [30]. The ideas of [30, 31, 34] are to prove that positive radial solutions are non-degenerate. We refer the reader to $[30,31,34]$ for the precise meaning of non-degenerate solutions.

In the general case $1<p<N$, among other results, Adimurthi and Yadava [1] proved the uniqueness of positive radial solutions to the following prototype of equation (1.1)

$$
\begin{cases}-\Delta_{p} u=|u|^{p^{*}-2} u+\lambda|u|^{p-2} u & \text { in } B \\ u=0 & \text { on } \partial B\end{cases}
$$

where $\lambda \in \mathbf{R}$. The approach of Adimurthi and Yadava [1], roughly speaking, is as follows: Suppose that $u$ and $v$ are two positive radial solutions to equation (1.12). If $u \geq v$ or $v \geq u$ in $B$, then it can be proved easily that $u \equiv v$ in $B$. If $u \not \equiv v$ in $B$, then $u / v$ is a positive continuous function on $\bar{B}$, the closure of $B$. Then $0<\min _{\bar{B}}(u / v)<1$ and $0<\min _{\bar{B}}(v / u)<1$. They excluded both cases by virtue of a generalized Pohozaev-type identity from Ni and Serrin $[26,27]$ or Pucci and Serrin [29].

In the present paper, we use the idea of Adimurthi and Yadava [1]. We obtain the following uniqueness result. 
Theorem 1.2. Assume that $1<p<N,-\infty<\mu<\bar{\mu}=((N-p) / p)^{p}$ and $0 \leq s<p$. If $\lambda \leq 0$, then equation (1.1) admits no positive radial solution in $B$. If $\lambda>0$, then equation (1.1) admits at most one positive radial solution in $B$.

We remark that in the case $p=2,0<\mu<\bar{\mu}$ and $s=0$, that is, the case considered by Ramaswamy and Santra [30], a nondegeneracy argument for uniqueness was applied. It seems difficult to extend their argument to the general case when $p \neq 2$, since there is no transform that can be used as that of Ramaswamy and Santra [30]. In this sense, we obtain a direct and different proof from that of Ramaswamy and Santra [30].

The idea of Adimurthi and Yadava [1] also does not work for the general case $\mu \neq 0$ in a direct way, since when $\mu=0$ all positive solutions to equation (1.1) are bounded, while it is not the case when $\mu>0$. However, a further study on the equation (1.1) shows that positive solutions to equation (1.1) behaves in a uniform way in the neighborhood of the origin, see Theorem 1.3 below. This fact implies the possibility to apply the idea of Adimurthi and Yadava [1]. Indeed, to apply the idea of Adimurthi and Yadava [1], first we establish a generalized Pohozaev-type identity for solutions to equation (1.1). This is done by combining the generalized Pohozaev-type identity $[26,27,29]$ together with some apriori estimates on positive radial solutions to equation (1.1). Then we show that $u / v$ is a positive continuous function on $\bar{B}$, if $u$ and $v$ are two positive radial solutions to equation (1.1). This is done by a precise estimate on the asymptotic behavior of $u(x)$ and $v(x)$ as $x \rightarrow 0$. Finally, we prove that $u \equiv v$ in the same way as that of Adimurthi and Yadava [1]. Therefore the following estimates on the asymptotic behavior of positive radial solutions to equation (1.1) play a key role in our argument.

Theorem 1.3. Assume that $1<p<N,-\infty<\mu<\bar{\mu}=((N-p) / p)^{p}, 0 \leq s<p$ and $\lambda \in \mathbf{R}$. Let $u \in W_{0}^{1, p}(B)$ be a positive radial solution to equation (1.1). There exists a constant $C>0$ such that

$$
\lim _{|x| \rightarrow 0} u(x)|x|^{\gamma_{1}}=C \quad \text { and } \quad \lim _{|x| \rightarrow 0}|\nabla u(x)||x|^{\gamma_{1}+1}=\left|\gamma_{1}\right| C .
$$

Here $\gamma_{1}$ is defined as in (1.9).

1.2. Classification of positive radial weak solutions to equation (1.10). Now we move to equation (1.10). Note that equation (1.10) is invariant under the dilation

$$
u^{\tau}(x)=\tau^{\frac{N-p}{p}}(\tau x)
$$

for $\tau>0$. That is, if $u$ is a solution to equation (1.10), then so is $u^{\tau}$. In the case $\mu=s=0$, equation (1.10) is also invariant under translations. Taking into account the invariance of equation (1.10) with respect to (1.13), we are concerned with the classification of positive radial solutions (with respect to the origin) to equation (1.10) in the Sobolev space $\mathcal{D}^{1, p}\left(\mathbf{R}^{N}\right)$.

In many cases, exact forms of positive radial solutions to equation (1.10) in $\mathcal{D}^{1, p}\left(\mathbf{R}^{N}\right)$ are known. When $p=2$, equation (1.10) is reduced to

$$
-\Delta u-\frac{\mu}{|x|^{2}} u=\frac{|u|^{2^{*}(s)-2} u}{|x|^{s}} \text { in } \mathbf{R}^{N} \text {. }
$$

Assume that $-\infty<\mu<\bar{\mu}$ and $0 \leq s<2$. By Proposition 2.6 and Proposition 8.1 of Catrina and Wang [10], every positive radial solution $u \in \mathcal{D}^{1,2}\left(\mathbf{R}^{N}\right)$ to equation 
(1.14) are of the form

$$
u(x)=U_{2, \mu, s}^{\tau}(x)=\tau^{(N-2) / 2} U_{2, \mu, s}(\tau x),
$$

for $\tau>0$, where

$$
U_{2, \mu, s}(x)=c_{2, \mu, s}\left(|x|^{\frac{2-s}{2}\left(1-\nu_{\mu}\right)}+|x|^{\frac{2-s}{2}\left(1+\nu_{\mu}\right)}\right)^{-\frac{N-2}{2-s}}
$$

with

$$
\nu_{\mu}=\sqrt{1-\frac{\mu}{\bar{\mu}}} \quad \text { and } \quad c_{2, \mu, s}=\left(\frac{4(N-s)(\bar{\mu}-\mu)}{N-2}\right)^{\frac{N-2}{2(2-s)}} .
$$

For some special cases of equation (1.14), the explicit formula of $U_{2, \mu, s}$ was also obtained by many other authors. We refer the reader to Aubin [2] and Talenti [32] for the case $\mu=s=0$, Lieb [24] for the case $\mu=0$ and $0 \leq s<2$, Terracini [33] for the case $0 \leq \mu<\bar{\mu}$ and $s=0$, and Chou and Chu [11] for the case $0 \leq \mu<\bar{\mu}$ and $0 \leq s<2$.

When $1<p<N$ and $\mu=0$, equation (1.10) is reduced to

$$
-\Delta_{p} u=\frac{|u|^{p^{*}(s)-2} u}{|x|^{s}} \text { in } \mathbf{R}^{N} \text {. }
$$

Ghoussoub and Yuan [17] proved that all positive radial solutions $u \in \mathcal{D}^{1, p}\left(\mathbf{R}^{N}\right)$ to equation (1.15) are of the form

$$
u(x)=U_{p, 0, s}^{\tau}(x)=\tau^{(N-p) / p} U_{p, 0, s}(\tau x)
$$

for $\tau>0$ (when $s=0$, it is also invariant with respect to translations), where

$$
U_{p, 0, s}(x)=c_{p, 0, s}\left(1+|x|^{\frac{p-s}{p-1}}\right)^{-\frac{N-p}{p-s}}
$$

with

$$
c_{p, 0, s}=\left((N-s)\left(\frac{N-p}{p-1}\right)^{p-1}\right)^{\frac{N-p}{p(p-s)}} .
$$

In the case when $s=0$, above exact form was also obtained by Guedda and Véron [15].

In the general case when $1<p<N,-\infty<\mu<\bar{\mu}(\mu \neq 0)$ and $0 \leq s<p$, the exact form for positive radial solutions to equation (1.10) in $\mathcal{D}^{1, p}\left(\mathbf{R}^{N}\right)$ seems to be unknown. In the particular case $1<p<N, 0<\mu<\bar{\mu}$ and $s=0$, that is, consider equation (1.5). Abdellaoui, Felli and Peral [3] proved the uniqueness of positive radial solutions to equation (1.5) in $\mathcal{D}^{1, p}\left(\mathbf{R}^{N}\right)$, up to a dilation (1.13). Moreover, they showed that if $u \in \mathcal{D}^{1, p}\left(\mathbf{R}^{N}\right)$ is a positive radial solution to equation (1.5), then $u$ satisfies the estimates (1.6) and (1.7).

In this paper, we follow the argument of Abdellaoui, Felli and Peral [3] and extend their uniqueness result to the general case. We obtain the following result.

Theorem 1.4. Assume that $1<p<N,-\infty<\mu<\bar{\mu}=((N-p) / p)^{p}$ and $0 \leq s<p$. Then up to a dilation (1.13), there exists at most one positive radial solution $u \in \mathcal{D}^{1, p}\left(\mathbf{R}^{N}\right)$ to equation (1.10). Moreover, $u$ satisfies the estimates (1.6) and (1.7).

The paper is organized as follows. We prove Theorem 1.2 in Section 2 and Theorem 1.4 in Section 3. The proof of Theorem 1.3 is routine and given in Section 4. Some preliminary results are given in the Appendixes. 
With no loss of generality, we assume throughout this paper that $B$ is the unit ball centered at the origin. By abuse of notation, we write $u(x)=u(r)$ with $r=|x|$, whenever $u$ is a radial function.

\section{Proof of Theorem 1.2}

In this section we prove Theorem 1.2. The following Pohozaev-type identity will be used in the proof of Theorem 1.2.

Lemma 2.1. Let $u \in W_{0}^{1, p}(B)$ be a positive radial solution to equation (1.1). Then for any $0<r \leq 1$ we have

$$
\begin{aligned}
\lambda \int_{0}^{r} u(t)^{p} t^{N-1} d t= & \frac{p-1}{p}\left|u^{\prime}(r)\right|^{p} r^{N}+\frac{N-p}{p} u(r)\left|u^{\prime}(r)\right|^{p-2} u^{\prime}(r) r^{N-1} \\
& +\frac{1}{p} u(r)^{p}\left(\mu r^{N-p}+\lambda r^{N}\right)+\frac{1}{p^{*}(s)} u(r)^{p^{*}(s)} r^{N-s} .
\end{aligned}
$$

Proof. For any $0<a<r \leq 1$, we have from the Pohozaev-type variational identity of Ni and Serrin [26, 27] or Pucci and Serrin [29] that

$$
\begin{aligned}
\lambda \int_{a}^{r} u(t)^{p} t^{N-1} d t= & \frac{p-1}{p}\left(\left|u^{\prime}(r)\right|^{p} r^{N}-\left|u^{\prime}(a)\right|^{p} a^{N}\right)+\frac{\mu}{p}\left(u(r)^{p} r^{N-p}-u(a)^{p} a^{N-p}\right) \\
& +\frac{N-p}{p}\left(u(r)\left|u^{\prime}(r)\right|^{p-2} u^{\prime}(r) r^{N-1}-u(a)\left|u^{\prime}(a)\right|^{p-2} u^{\prime}(a) a^{N-1}\right) \\
& +\left(\frac{1}{p^{*}(s)} \frac{u(r)^{p^{*}(s)}}{r^{s}}+\frac{\lambda}{p} u(r)^{p}\right) r^{N}-\left(\frac{1}{p^{*}(s)} \frac{u(a)^{p^{*}(s)}}{a^{s}}+\frac{\lambda}{p} u(a)^{p}\right) a^{N} .
\end{aligned}
$$

By Theorem 1.3 and the fact that $\gamma_{1}<(N-p) / p$, we obtain $(2.1)$ by sending $a \rightarrow 0$ in above equality.

We start the proof of Theorem 1.2 with the following result.

Proposition 2.2. Assume that $\lambda>0$. If $u, v \in W_{0}^{1, p}(B)$ are two positive radial solutions to equation (1.1) and $u \geq v$ in $B$, then $u \equiv v$ in $B$.

Proof. Suppose that $u, v \in W_{0}^{1, p}(B)$ are two positive radial solutions to equation (1.1) and $u \geq v$ in $B$. Set $\rho_{1}=u^{p^{*}(s)-p} r^{-s}+\lambda$ and $\rho_{2}=v^{p^{*}(s)-p} r^{-s}+\lambda$. Then $\rho_{1} \geq \rho_{2}>0$ in $B$ by assumptions. Applying Lemma A.1, we deduce that $u=v=0$ on the set $\left\{x \in B: \rho_{1}(x)>\rho_{2}(x)\right\}$. Since $u, v$ are positive functions, we have that

$$
\left\{x \in B: \rho_{1}(x)>\rho_{2}(x)\right\}=\emptyset .
$$

That is, $\rho_{1} \equiv \rho_{2}$ in $B$. The proof of Proposition 2.2 is complete.

We also need the following lemma.

Lemma 2.3. Assume that $1<p<N$. Let $v$ be a positive solution to equation

$$
\left\{\begin{array}{l}
-\left(r^{N-1}\left|v^{\prime}(r)\right|^{p-2} v^{\prime}(r)\right)^{\prime}=g(r, v(r)) r^{N-1} \quad \text { for } \frac{1}{2}<r<1 \\
v(1)=0,
\end{array}\right.
$$

where $g:[1 / 2,1] \times[0, \infty) \rightarrow \mathbf{R}$ is a function satisfying that

$$
|g(r, t)| \leq C_{0} t^{p-1} \quad \text { for }(r, t) \in\left(\frac{1}{2}, 1\right) \times(0, \infty) .
$$

Then

$$
v^{\prime}(1)<0
$$


Proof. This lemma should be well known. But as we did not find a proper reference, we give a proof here for completeness. Since $v$ is a positive solution and $v(1)=0$, then $v^{\prime}(1) \leq 0$. Suppose that Lemma 2.3 is not true. That is, we suppose that

$$
v^{\prime}(1)=0 .
$$

Integrate each side of equation (2.2) from $r$ to 1 . We obtain, by (2.4), that

$$
\begin{aligned}
r^{N-1}\left|v^{\prime}(r)\right|^{p-2} v^{\prime}(r) & =\int_{r}^{1} g(\tau, v(\tau)) \tau^{N-1} d \tau \leq C_{0} \int_{r}^{1} v(\tau)^{p-1} d \tau \\
& \leq C_{0}(1-r)^{\frac{1}{p}}\left(\int_{r}^{1} v(\tau)^{p} d \tau\right)^{\frac{p-1}{p}}
\end{aligned}
$$

for all $1 / 2 \leq r<1$. It follows from above that

$$
\left|v^{\prime}(r)\right|^{p} \leq C(1-r)^{\frac{1}{p-1}} \int_{r}^{1} v(\tau)^{p} d \tau
$$

for all $1 / 2 \leq r<1$. Combine Hölder's inequality and the assumption that $v(1)=0$. We obtain that

$$
\begin{aligned}
v(r)^{p} & =-p \int_{r}^{1} v(\tau)^{p-1} v^{\prime}(\tau) d \tau \leq C\left(\int_{r}^{1} v(\tau)^{p} d \tau\right)^{\frac{p-1}{p}}\left(\int_{r}^{1}\left|v^{\prime}(\tau)\right|^{p} d \tau\right)^{\frac{1}{p}} \\
& \leq C(1-r)^{\frac{1}{p-1}} \int_{r}^{1} v(\tau)^{p} d \tau
\end{aligned}
$$

for all $1 / 2 \leq r<1$. Define $w(t)=v(1-t)$ for $0 \leq t \leq 1 / 2$. Above equality is equivalent to

$$
w(t)^{p} \leq C t^{\frac{1}{p-1}} \int_{0}^{t} w(\tau)^{p} d \tau
$$

for all $0 \leq t \leq 1 / 2$. Note that $w(0)=0$. It follows from the Gronwall's inequality that

which is equivalent to

$$
w(t)=0 \quad \text { for all } 0 \leq t \leq \frac{1}{2},
$$

$$
v(r)=0 \text { for all } \frac{1}{2} \leq r \leq 1 .
$$

We reach a contradiction, as we assume that $v$ is positive. The proof of Lemma 2.3 is complete.

Now we prove Theorem 1.2.

Proof of Theorem 1.2. We have two cases: Case 1: $\lambda \leq 0$. Case 2: $\lambda>0$.

Consider Case 1. Suppose that $u \in W_{0}^{1, p}(B)$ is a positive radial solution to equation (1.1). Then $u$ satisfies the identity (2.1). Take $r=1$ in (2.1). We obtain that

$$
0 \geq \lambda \int_{0}^{1} u(t)^{p} t^{N-1} d t=\frac{p-1}{p}\left|u^{\prime}(1)\right|^{p} \geq 0 .
$$

If $\lambda<0$, then $u \equiv 0$ in $B$ by (2.5). We obtain a contradiction. If $\lambda=0$, then $u^{\prime}(1)=0$ by $(2.5)$. Note that $u(1)=0$. Apply Lemma 2.3 to $u$ with $g(r, u)$ given by

$$
g(r, u)=\left(\mu r^{-p}+u^{p^{*}(s)-p} r^{-s}+\lambda\right) u^{p-1} .
$$


We obtain that $u(r) \equiv 0$ for $1 / 2 \leq r \leq 1$. We reach a contradiction. Hence in Case 1 , that is, $\lambda \leq 0$, there has no positive radial solution to equation (1.1) in $B$.

We remark that when $\mu=0$, Adimurthi and Yanava [1] pointed out that an observation of Knaap and Peletier [22] implies that $u(r) \equiv 0$ for $0<r \leq 1$. They also pointed out that a more general theorem given by Franchi, Lanconelli and Serrin [12] also claims that $u(r) \equiv 0$ for $0<r \leq 1$.

Consider Case 2. Suppose that $u, v \in W_{0}^{1, p}(B)$ are two positive radial solutions to equation (1.1). We prove that $u \equiv v$ in $B$. Suppose, on the contrary, that $u \not \equiv v$ in $B$. That is,

$$
u(r) \not \equiv v(r) \quad \text { for } 0<r<1
$$

Let

$$
w(r)=\frac{u(r)}{v(r)} \quad \text { for } 0<r<1 .
$$

Then $w$ is a positive continuous function in $(0,1)$.

First, we claim that $w$ can be extended to $r=0$ and $r=1$ such that $w$ is a positive continuous function on $[0,1]$. Indeed, by Theorem 1.3 , there exist constants $C_{u}, C_{v}>0$ such that

$$
\lim _{r \rightarrow 0} u(r) r^{\gamma_{1}}=C_{u} \quad \text { and } \quad \lim _{r \rightarrow 0} v(r) r^{\gamma_{1}}=C_{v} .
$$

Then we have that

$$
\lim _{r \rightarrow 0} w(r)=\frac{C_{u}}{C_{v}}>0 .
$$

Thus we can extend $w$ continuously to $r=0$ by setting $w(0)=C_{u} / C_{v}$. On the other hand, by L'Hospital's rule, we have that

$$
\lim _{r \rightarrow 1} w(r)=\frac{u^{\prime}(1)}{v^{\prime}(1)}>0
$$

since both $u^{\prime}(1)$ and $v^{\prime}(1)$ are negative by Lemma 2.3. Hence we can extend $w$ continuously to $r=1$ by setting $w(1)=u^{\prime}(1) / v^{\prime}(1)$. Then $w$ is a positive continuous function on $[0,1]$.

Next, set

$$
\alpha=\inf _{r \in[0,1]} w(r) .
$$

Then $\alpha>0$. We claim that $\alpha<1$. Otherwise, if $\alpha \geq 1$, then $u \geq v$ in $(0,1)$. Proposition 2.2 implies that $u \equiv v$ in $(0,1)$. This contradicts to $(2.6)$. Hence $0<$ $\alpha<1$. Since $w$ is continuous on $[0,1], \alpha$ can be achieved by $w$ on $[0,1]$. Let $r_{\alpha}$ be such that

We claim that

$$
r_{\alpha}=\inf \{t \in[0,1]: w(t)=\alpha\}
$$

$$
r_{\alpha}=0 \text {. }
$$

Otherwise, we have $0<r_{\alpha} \leq 1$. If $r_{\alpha}=1$, that is, $w(1)=\alpha$ and $w(r)>\alpha$ for $0 \leq r<1$. Then we deduce that $u^{\prime}(1)=\alpha v^{\prime}(1)$, and $u(r)>\alpha v(r)$ for $0 \leq r<1$. Take $r=r_{\alpha}=1$ in (2.1). Since both $u, v$ satisfy (2.1), we obtain that

$$
0<\lambda \int_{0}^{1}\left(u(t)^{p}-\alpha^{p} v(t)^{p}\right) t^{N-1} d t=\frac{p-1}{p}\left(\left|u^{\prime}(1)\right|^{p}-\alpha^{p}\left|v^{\prime}(1)\right|^{p}\right)=0 .
$$


We reach a contradiction. If $0<r_{\alpha}<1$, then $w\left(r_{\alpha}\right)=\alpha$ and $w(r)>\alpha$ for $0 \leq r<r_{\alpha}$. Note that $w^{\prime}\left(r_{\alpha}\right)=0$. We deduce that $u>\alpha v$ in $\left(0, r_{\alpha}\right), u\left(r_{\alpha}\right)=\alpha v\left(r_{\alpha}\right)$ and $u^{\prime}\left(r_{\alpha}\right)=\alpha v^{\prime}\left(r_{\alpha}\right)$. Take $r=r_{\alpha}$ in (2.1). We obtain that

$$
\begin{aligned}
0 & <\lambda \int_{0}^{r_{\alpha}}\left(u(t)^{p}-\alpha^{p} v(t)^{p}\right) t^{N-1} d t=\frac{r_{\alpha}^{N-s}}{p^{*}(s)}\left(u\left(r_{\alpha}\right)^{p^{*}(s)}-\alpha^{p} v\left(r_{\alpha}\right)^{p^{*}(s)}\right) \\
& =\frac{r_{\alpha}^{N-s}}{p^{*}(s)}\left(\alpha^{p^{*}(s)}-\alpha^{p}\right) v\left(r_{\alpha}\right)^{p^{*}(s)}<0,
\end{aligned}
$$

since $0<\alpha<1$ and $p^{*}(s)>p$. We reach a contradiction. This proves $(2.7)$.

Therefore we obtain that $w(0)=\alpha<1$. Recall that $w(0)=C_{u} / C_{v}$. Hence

$$
C_{u}<C_{v} \text {. }
$$

Similarly, consider $\tilde{w}(r)=v(r) / u(r)$. Repeat above procedure with respect to $\tilde{w}(r)$. We obtain that $C_{v} / C_{u}=\tilde{w}(0)<1$. Hence

$$
C_{v}<C_{u}
$$

We reach a contradiction. Therefore $u \equiv v$ in $(0,1)$. The proof of Theorem 1.2 is complete.

\section{Proof of Theorem 1.4}

In this section we prove Theorem 1.4. Before giving the proof of Theorem 1.4, let us revisit the following prototype of equation (1.10)

$$
-\Delta u=|u|^{2^{*}-2} u \text { in } \mathbf{R}^{N},
$$

where $2^{*}=2 N /(N-2)$ and $N \geq 3$. Let $u \in \mathcal{D}^{1,2}\left(\mathbf{R}^{N}\right)$ be a positive radial solution to equation (3.1). Applying the transform

$$
t=\log r \quad \text { and } \quad \psi(t)=r^{\frac{N-2}{2}} u(r),
$$

for $r \in(0, \infty)$, we deduce that

$$
\psi^{\prime \prime}(t)-\left(\frac{N-2}{2}\right)^{2} \psi(t)+\psi^{2^{*}-1}(t)=0 \quad \text { in } \mathbf{R} .
$$

Solving equation (3.3) (see details in e.g. [5, Section 1]) and taking into account that $u \in \mathcal{D}^{1,2}\left(\mathbf{R}^{N}\right)$, we obtain that

$$
u(x)=\left(\frac{\lambda \sqrt{N(N-2)}}{\lambda^{2}+\left|x-x_{0}\right|^{2}}\right)^{\frac{N-2}{2}}, \quad \lambda>0, x_{0} \in \mathbf{R}^{N} .
$$

So this gives the exact form of $u$.

In above approach the transform (3.2) turns equation (3.1) into the ordinary differential equation (3.3) which can be solved explicitly. In the general case $1<p<$ $N,-\infty<\mu<\bar{\mu}$ and $0 \leq s<p$, a similar type of transform to (3.2) will be used to turn equation (1.10) into an ordinary differential equation system. Then we follow the argument of Abdellaoui, Felli and Peral [3] to establish the uniqueness (up to a dilation) of positive radial weak solutions to equation (1.10).

Let $u \in \mathcal{D}^{1, p}\left(\mathbf{R}^{N}\right)$ be a positive radial solution to equation (1.10). Then we have

$$
\int_{0}^{\infty}\left(\frac{|u(r)|^{p^{*}(s)}}{r^{s}}+\left|u^{\prime}(r)\right|^{p}\right) r^{N-1} d r=\frac{1}{\omega_{N-1}} \int_{\mathbf{R}^{N}}\left(\frac{|u|^{p^{*}(s)}}{|x|^{s}}+|\nabla u|^{p}\right) d x<\infty
$$


where $\omega_{N-1}$ is the surface measure of the unit sphere in $\mathbf{R}^{N}$, and $u(x)=u(r)$ with $r=|x|$ solves the equation

$$
\begin{cases}-\left(r^{N-1}\left|u^{\prime}(r)\right|^{p-2} u^{\prime}(r)\right)^{\prime}=\left(\frac{\mu}{r^{p}}+\frac{u(r)^{p^{*}(s)-p}}{r^{s}}\right) u(r)^{p-1} r^{N-1}, & r \in(0, \infty), \\ u(r)>0, & r \in(0, \infty) .\end{cases}
$$

Apply the transform:

$$
t=\log r, \quad y(t)=r^{\delta} u(r), \quad z(t)=r^{(p-1)(\delta+1)}\left|u^{\prime}(r)\right|^{p-2} u^{\prime}(r),
$$

where we denote $\delta=(N-p) / p$ in this section. We obtain by equation (3.5) that $y$ satisfies

$$
y^{\prime}=\delta y+|z|^{\frac{1}{p-1}-1} z, \quad y>0 \quad \text { in } \mathbf{R},
$$

and $z$ satisfies

$$
z^{\prime}=-\delta z-y^{p^{*}(s)-1}-\mu y^{p-1} \quad \text { in } \mathbf{R} .
$$

Define $V: \mathbf{R}^{2} \rightarrow \mathbf{R}$ by

$$
V(a, b)=\frac{1}{p^{*}(s)}|a|^{p^{*}(s)}+\frac{\mu}{p}|a|^{p}+\delta a b+\frac{1}{p^{\prime}}|b|^{p^{\prime}} .
$$

Here $p^{\prime}=p /(p-1)$. It follows from equations (3.7) and (3.8) that

$$
\frac{d}{d t}(V(y(t), z(t)))=0, \quad \forall t \in \mathbf{R} .
$$

Hence there is a constant $K$ such that

$$
V(y(t), z(t)) \equiv K, \quad \forall t \in \mathbf{R} .
$$

Since $u \in \mathcal{D}^{1, p}\left(\mathbf{R}^{N}\right)$ is a radial function, we have (see [25, Corollary II.1] and its proof)

Thus

$$
\lim _{r \rightarrow 0} r^{\delta} u(r)=\lim _{r \rightarrow \infty} r^{\delta} u(r)=0
$$

$$
\lim _{|t| \rightarrow \infty} y(t)=0
$$

Note also that by (3.4) we have

$$
\liminf _{r \rightarrow 0} r^{\delta+1}\left|u^{\prime}(r)\right|=\liminf _{r \rightarrow \infty} r^{\delta+1}\left|u^{\prime}(r)\right|=0 .
$$

Hence

$$
\liminf _{|t| \rightarrow \infty}|z(t)|=0 .
$$

Sending $|t| \rightarrow \infty$ in (3.10), we deduce that $K=0$, that is,

$$
\frac{1}{p^{*}(s)} y(t)^{p^{*}(s)}+\frac{\mu}{p} y(t)^{p}+\delta y(t) z(t)+\frac{1}{p^{\prime}}|z(t)|^{p^{\prime}}=0, \quad \forall t \in \mathbf{R} .
$$

We claim that $y$ is bounded on $\mathbf{R}$. Precisely, set

$$
M=\left(\frac{p^{*}(s)(\bar{\mu}-\mu)}{p}\right)^{\frac{1}{p^{*}(s)-p}} .
$$

Lemma 3.1. We have

$$
y(t) \leq M, \quad \forall t \in \mathbf{R} .
$$

Moreover, $y\left(t_{0}\right)=M$ at a point $t_{0} \in \mathbf{R}$ if and only if $\delta y\left(t_{0}\right)=-\left|z\left(t_{0}\right)\right|^{\frac{1}{p-1}-1} z\left(t_{0}\right)$. 
Proof. Recall that Young's inequality gives that

$$
a b \leq \frac{1}{p}|a|^{p}+\frac{1}{p^{\prime}}|b|^{p^{\prime}}, \quad \forall a, b \in \mathbf{R},
$$

and the equality holds if and only if $|a|=|b|^{\frac{1}{p-1}}$ and $a b \geq 0$. Hence

$$
-\delta y(t) z(t) \leq \frac{\delta^{p}}{p} y(t)^{p}+\frac{1}{p^{\prime}}|z(t)|^{p^{\prime}}, \quad \forall t \in \mathbf{R},
$$

and the equality holds at some $t=t_{0}$ if and only if $\delta y\left(t_{0}\right)=\left|z\left(t_{0}\right)\right|^{\frac{1}{p-1}}$ and $z\left(t_{0}\right)<0$. Note that $\delta^{p}=\bar{\mu}=((N-p) / p)^{p}$. Combining (3.14) and (3.11) gives us that

$$
\frac{1}{p^{*}(s)} y(t)^{p^{*}(s)} \leq \frac{\bar{\mu}-\mu}{p} y(t)^{p},
$$

which implies (3.13), and the equality holds at $t=t_{0} \in \mathbf{R}$ if and only if $\delta y\left(t_{0}\right)=$ $-\left|z\left(t_{0}\right)\right|^{\frac{1}{p-1}-1} z\left(t_{0}\right)$. This proves the lemma.

Since $y$ is continuous in $\mathbf{R}$ and $y(t) \rightarrow 0$ as $|t| \rightarrow \infty, y$ achieves its maximum in $\mathbf{R}$. Let $t_{0} \in \mathbf{R}$ be such that $y\left(t_{0}\right)=\max _{\mathbf{R}} y$. Then $t_{0}$ is a critical point of $y$, that is, $y^{\prime}\left(t_{0}\right)=0$. By equation (3.7), we obtain that $\delta y\left(t_{0}\right)=-\left|z\left(t_{0}\right)\right|^{\frac{1}{p-1}-1} z\left(t_{0}\right)$. Then Lemma 3.1 implies that $y\left(t_{0}\right)=M$. We claim that $t_{0}$ is the unique critical point of $y$ in $\mathbf{R}$. Indeed, suppose that $t_{1} \in \mathbf{R}$ is another critical point of $y$. Then combining equation (3.7) and Lemma 3.1 yields that $y\left(t_{1}\right)=M$. With no loss of generality, we assume that $t_{1}<t_{0}$. We prove that $y \equiv M$ in $\left[t_{1}, t_{0}\right]$. Otherwise, there exists $t_{2} \in\left(t_{1}, t_{0}\right)$ such that $y\left(t_{2}\right)=\min _{\left[t_{1}, t_{0}\right]} y<M$. Then $y^{\prime}\left(t_{2}\right)=0$. Combining equation (3.7) and Lemma 3.1 again yields that $y\left(t_{2}\right)=M$. We reach a contradiction. Hence $y \equiv M$ on $\left[t_{1}, t_{0}\right]$. But then we have $y^{\prime} \equiv 0$ on $\left[t_{1}, t_{0}\right]$. Consider equation (3.7) on the interval $\left[t_{1}, t_{0}\right]$. We obtain that $z \equiv-(\delta M)^{p-1}$ on $\left[t_{1}, t_{0}\right]$. Then we derive from equation (3.8) that

$$
\delta(\delta M)^{p-1}-M^{p^{*}(s)-1}-\mu M^{p-1}=0,
$$

which implies that $M=(\bar{\mu}-\mu)^{\frac{1}{p^{*}(s)-p}}$. We reach a contradiction to (3.12). Hence $t_{0}$ is the unique critical point of $y$ in $\mathbf{R}$. Thus $y^{\prime}(t)>0$ for $t<t_{0}$ and $y^{\prime}(t)<0$ for $t>t_{0}$. Note that both equations (3.7) and (3.8) are invariant under translations. Therefore, up to a translation, we assume in the rest of this section that $y$ satisfies

$$
\left\{\begin{array}{l}
y(0)=\max _{t \in \mathbf{R}} y=M, \text { and } \\
y^{\prime}>0 \text { in }(-\infty, 0) \text { and } y^{\prime}<0 \text { in }(0, \infty) .
\end{array}\right.
$$

It follows immediately from equation (3.7) and (3.15) that

$$
z(0)=-(\delta M)^{p-1} .
$$

Lemma 3.2. For the function $z$, we have,

(1) $z$ is a bounded continuous function on $\mathbf{R}$;

(2) in the case $0 \leq \mu<\bar{\mu}, z(t)<0$ for all $t \in \mathbf{R}$;

(3) in the case $\mu<0$, there exists a unique point $t_{-} \in \mathbf{R}, t_{-}<0$, such that $z>0$ in $\left(-\infty, t_{-}\right)$and $z<0$ in $\left(t_{-}, \infty\right)$.

Proof. (1) The boundedness of $z$ follows from (3.11) and boundedness of $y$.

(2) In the case $0 \leq \mu<\bar{\mu}$, it follows from (3.11) easily that $z(t)<0$ for all $t \in \mathbf{R}$.

(3) Consider the case $\mu<0$. We claim that there exists a constant $L>0$ sufficiently large such that $z(t)>0$ for $t<-L$. Indeed, since $z$ is proved to be 
bounded on $\mathbf{R}$, we have $e^{\delta t} z(t) \rightarrow 0$ as $t \rightarrow-\infty$. Solve equation (3.8). We deduce that

$$
e^{\delta t} z(t)=\int_{-\infty}^{t} e^{\delta s} y(s)^{p-1}\left(-\mu-y(s)^{p^{*}(s)-p}\right) d s \quad \text { for } t \in \mathbf{R} .
$$

Since $\mu<0$ and $y(t) \rightarrow 0$ as $t \rightarrow-\infty$, there exists $L>0$ sufficiently large such that $-\mu-y(s)^{p^{*}(s)-p}>0$ for $s<-L$. Thus $e^{\delta t} z(t)>0$ for $t<-L$. This proves the claim.

Note that by $(3.16)$ we have $z(0)<0$. Hence, by above claim, the set

$$
Z=\{t \in \mathbf{R}: z(t)=0\}
$$

is not empty. To prove (3), it is enough to prove that $Z$ consists of only one point. Let $t_{0} \in \mathbf{R}$ be an arbitrary point in $Z$. Then $z\left(t_{0}\right)=0$. We show that $t_{0}$ can be uniquely determined. Substitute $t=t_{0}$ into equation (3.7). We obtain that $y^{\prime}\left(t_{0}\right)=\delta y\left(t_{0}\right)>0$. Hence $t_{0} \in(-\infty, 0)$ holds by (3.15). Substitute $t=t_{0}$ into equation (3.11). We obtain that

$$
y\left(t_{0}\right)=\left(-p^{*}(s) \mu / p\right)^{1 /\left(p^{*}(s)-p\right)} .
$$

Since $y$ is strictly monotone in $(-\infty, 0)$ by $(3.15)$, we find that $t_{0}$ is the unique point in $(-\infty, 0)$ which satisfies $(3.17)$. This proves that $Z$ consists of only one point. Denote by $t_{-}$the point in $Z$. The proof of (3) is complete.

Now we study the asymptotic behaviors of $y$ and $z$. Let $\gamma \in \mathbf{R}$ be an arbitrary number and define

$$
y_{\gamma}(t)=e^{(\gamma-\delta) t} y(t), \quad t \in \mathbf{R} .
$$

By (3.7) and (3.15), we have

$$
\left\{\begin{array}{l}
y_{\gamma}^{\prime}(t)=(\gamma-H(t)) y_{\gamma}(t), \quad t \in \mathbf{R} \\
y_{\gamma}(0)=M
\end{array}\right.
$$

where $M$ is defined as in (3.12) and $H: \mathbf{R} \rightarrow \mathbf{R}$ is defined by

$$
H(t)=-\frac{|z(t)|^{\frac{1}{p-1}-1} z(t)}{y(t)}, \quad t \in \mathbf{R} .
$$

Note that $H$ is a continuous function on $\mathbf{R}$. Let $t_{-}$be the number defined as in Lemma 3.2 in the case $\mu<0$. $H$ is continuously differentiable on $\mathbf{R}$ except at the point $t=t_{-}$in the case $\mu<0$.

The function $H$ plays a key role in the proof of Theorem 1.4. We derive the equation satisfied by $H$. For $t \neq t_{-}$, We have that

$$
\begin{aligned}
H^{\prime} & =y^{-2}\left(|z|^{\frac{1}{p-1}-1} z y^{\prime}-\frac{1}{p-1}|z|^{\frac{1}{p-1}-1} z^{\prime} y\right) \\
& =y^{-2}|z|^{\frac{2-p}{p-1}}\left(\left(\delta y+|z|^{\frac{1}{p-1}-1} z\right) z-\frac{1}{p-1} y\left(-\delta z-y^{p^{*}(s)-1}-\mu y^{p-1}\right)\right) \\
& =y^{-2}|z|^{\frac{2-p}{p-1}}\left(p^{\prime} \delta y z+|z|^{p^{\prime}}+\frac{1}{p-1} y^{p^{*}(s)}+\frac{\mu}{p-1} y^{p}\right) \\
& =p^{\prime} y^{-2}|z|^{\frac{2-p}{p-1}}\left(\delta y z+\frac{1}{p^{\prime}}|z|^{p^{\prime}}+\frac{1}{p} y^{p^{*}(s)}+\frac{\mu}{p} y^{p}\right) \\
& =\frac{p^{*}(s)-p}{p^{*}(s)(p-1)} y^{p^{*}(s)-2}|z|^{\frac{2-p}{p-1}}
\end{aligned}
$$


where the second equality follows from equations (3.7) and (3.8), and the last equality follows from (3.11). Thus by the definition (3.19) of $H$, we obtain that

$$
H^{\prime}(t)=\frac{p^{*}(s)-p}{p^{*}(s)(p-1)} y(t)^{p^{*}(s)-p}|H(t)|^{2-p} \quad \text { for } t \neq t_{-} .
$$

We remark that equation (3.20) holds at $t=t_{-}$if $0 \leq \mu$. On the other hand, by (3.11) we have that

$$
\frac{1}{p^{*}(s)} y^{p^{*}(s)-p}+\frac{1}{p^{\prime}} \frac{|z|^{p^{\prime}}}{y^{p}}+\delta \frac{z}{y^{p-1}}+\frac{\mu}{p} \equiv 0 \quad \text { in } \mathbf{R} .
$$

Recall that $\Gamma_{\mu}$ is defined as in (1.8). We obtain that

$$
\frac{p}{p^{*}(s)} y(t)^{p^{*}(s)-p}=-\Gamma_{\mu}(H(t)) \quad \text { in } \mathbf{R} \text {. }
$$

Combining equation (3.20) and equation (3.21) yields that

$$
H^{\prime}(t)=-\frac{p^{*}(s)-p}{p(p-1)}|H(t)|^{2-p} \Gamma_{\mu}(H(t)) \quad \text { for } t \neq t_{-} .
$$

That is, $H$ satisfies equation (3.22). We remark that when $0 \leq \mu<\bar{\mu}$, (3.22) holds for all $t \in \mathbf{R}$.

We claim that

$$
\lim _{t \rightarrow-\infty} H(t)=\gamma_{1} \quad \text { and } \quad \lim _{t \rightarrow \infty} H(t)=\gamma_{2}
$$

Indeed, Let $t \rightarrow-\infty$ and $t \rightarrow \infty$ in equation (3.21) respectively. we obtain that

$$
\lim _{t \rightarrow-\infty} \Gamma_{\mu}(H(t))=\lim _{t \rightarrow \infty} \Gamma_{\mu}(H(t))=0 .
$$

By equation (3.20), $H$ is strictly increasing in $\mathbf{R}$. Hence there exist $a, b,-\infty \leq a<$ $b \leq \infty$, such that $\lim _{t \rightarrow-\infty} H(t)=a$ and $\lim _{t \rightarrow \infty} H(t)=b$. Note that $\Gamma_{\mu}(\gamma) \rightarrow \infty$ as $|\gamma| \rightarrow \infty$. Hence (3.24) implies that $a, b$ are finite. Then $\Gamma_{\mu}(a)=\Gamma_{\mu}(b)=0$. Since $\gamma_{1}$ and $\gamma_{2}$ are the only two roots of $\Gamma_{\mu}$ in $\mathbf{R}$ and $\gamma_{1}<\gamma_{2}$, we obtain that $a=\gamma_{1}$ and $b=\gamma_{2}$, and then the claim is proved. Therefore, the monotonicity of $H$ implies that

$$
\gamma_{1}<H(t)<\gamma_{2}, \quad \forall t \in \mathbf{R} .
$$

Next we claim that

$$
\int_{-\infty}^{0}\left(H(s)-\gamma_{1}\right) d s+\int_{0}^{\infty}\left(\gamma_{2}-H(s)\right) d s<\infty .
$$

To this end, rewrite $\Gamma_{\mu}$ by $\Gamma_{\mu}(s)=\left(s-\gamma_{1}\right)\left(s-\gamma_{2}\right) \tilde{\Gamma}_{\mu}(s)$, where $\tilde{\Gamma}_{\mu}$ is a continuous function on $\mathbf{R}$ satisfying $\inf _{\mathbf{R}} \tilde{\Gamma}_{\mu}>0$. Then by change of variable, we have that

$$
\begin{aligned}
\int_{-\infty}^{2 t_{-}}\left(H(s)-\gamma_{1}\right) d s & =\int_{\gamma_{1}}^{H\left(2 t_{-}\right)} \frac{\left(\tau-\gamma_{1}\right) d \tau}{\tau^{\prime}(s)} \\
& =\int_{\gamma_{1}}^{H\left(2 t_{-}\right)} \frac{\left(\tau-\gamma_{1}\right) d \tau}{-\frac{p^{*}(s)-p}{p(p-1)}|\tau|^{2-p} \Gamma_{\mu}(\tau)} \\
& =\int_{\gamma_{1}}^{H\left(2 t_{-}\right)} \frac{p(p-1) d \tau}{\left(p^{*}(s)-p\right)|\tau|^{2-p}\left(\gamma_{2}-\tau\right) \tilde{\Gamma}_{\mu}(\tau)} \\
& \leq C \int_{\gamma_{1}}^{H\left(2 t_{-}\right)}|\tau|^{p-2} d \tau<\infty,
\end{aligned}
$$


where $C=p(p-1) /\left(\left(p^{*}(s)-p\right)\left(\gamma_{2}-H\left(2 t_{-}\right)\right) \inf _{\mathbf{R}} \tilde{\Gamma}_{\mu}\right)$. Similarly, we have that

$$
\begin{aligned}
\int_{0}^{\infty}\left(\gamma_{2}-H(s)\right) d s & =\int_{\delta}^{\gamma_{2}} \frac{\left(\gamma_{2}-\tau\right)}{\tau^{\prime}(s)} d \tau \\
& =\int_{\delta}^{\gamma_{2}} \frac{\left(\gamma_{2}-\tau\right) d \tau}{-\frac{p^{*}(s)-p}{p(p-1)}|\tau|^{2-p} \Gamma_{\mu}(\tau)} \\
& =\int_{\delta}^{\gamma_{2}} \frac{p(p-1) d \tau}{\left(p^{*}(s)-p\right)|\tau|^{2-p}\left(\tau-\gamma_{1}\right) \tilde{\Gamma}_{\mu}(\tau)} \\
& \leq C^{\prime} \int_{\delta}^{\gamma_{2}} \tau^{p-2} d \tau<\infty,
\end{aligned}
$$

where $C^{\prime}=p(p-1) /\left(\left(p^{*}(s)-p\right)\left(\delta-\gamma_{1}\right) \inf _{\mathbf{R}} \tilde{\Gamma}_{\mu}\right)$. This proves (3.26).

Now we are in a position to prove Theorem 1.4.

Proof of Theorem 1.4. Let $u \in \mathcal{D}^{1, p}\left(\mathbf{R}^{N}\right)$ be a positive radial solution to equation (1.10) and $(y, z)$ be defined as in (3.6) with respect to $u$. Let $H$ be defined as in (3.19). First we show that $u$ satisfies (1.6) and (1.7).

Integrate (3.18). We obtain that

$$
e^{(\gamma-\delta) t} y(t)=M \exp \left(\int_{0}^{t}(\gamma-H(\tau)) d \tau\right) \quad \text { for } t \in \mathbf{R} .
$$

Hence we derive that

$$
\left\{\begin{array}{l}
\lim _{t \rightarrow-\infty} e^{\left(\gamma_{1}-\delta\right) t} y(t)=M \exp \left(\int_{-\infty}^{0}\left(H-\gamma_{1}\right) d \tau\right)=: C_{1}, \quad \text { and } \\
\lim _{t \rightarrow \infty} e^{\left(\gamma_{2}-\delta\right) t} y(t)=M \exp \left(\int_{0}^{\infty}\left(\gamma_{2}-H\right) d \tau\right)=: C_{2},
\end{array}\right.
$$

which is equivalent to (1.6). Since

$$
|z(t)|^{\frac{1}{p-1}-1} z(t)=H(t) y(t)
$$

we derive from (3.23) and (3.28) that

$$
\left\{\begin{array}{l}
\lim _{t \rightarrow-\infty} e^{\left(\gamma_{1}-\delta\right) t}|z(t)|^{\frac{1}{p-1}}=C_{1}\left|\gamma_{1}\right|, \quad \text { and } \\
\lim _{t \rightarrow \infty} e^{\left(\gamma_{2}-\delta\right) t}|z(t)|^{\frac{1}{p-1}}=C_{2} \gamma_{2}
\end{array}\right.
$$

which is equivalent to (1.7). This proves that $u$ satisfies (1.6) and (1.7).

Next we prove the uniqueness of $u$ up to a dilation. Suppose that $u_{1}, u_{2} \in$ $\mathcal{D}^{1, p}\left(\mathbf{R}^{N}\right)$ are two positive radial solutions to equation (1.10). Define $\left(y_{i}, z_{i}\right)$ by the transform (3.6) with respect to $u_{i}$ for $i=1,2$. Define $H_{i}$ as in (3.19) with respect to $\left(y_{i}, z_{i}\right)$ for $i=1,2$. Then both $\left(y_{1}, z_{1}\right)$ and $\left(y_{2}, z_{2}\right)$ satisfy equations (3.7) and (3.8), and $H_{1}$ and $H_{2}$ satisfy equation (3.22).

To prove that $u_{1}=\lambda^{(p-N) / p} u_{2}(\cdot / \lambda)$ for some $\lambda>0$, it is equivalent to prove that $y_{1}=y_{2}\left(\cdot-t_{0}\right)$ for some $t_{0} \in \mathbf{R}$. Up to a translation, we assume that both $y_{1}$ and $y_{2}$ satisfy (3.15). We prove that $y_{1} \equiv y_{2}$ on $\mathbf{R}$. Note that under this assumption, we have that $\left(y_{1}(0), z_{1}(0)\right)=\left(y_{2}(0), z_{2}(0)\right)=\left(M,-(\delta M)^{p-1}\right)$ by $(3.15)$ and (3.16).

Define $f:\left(\gamma_{1}, \gamma_{2}\right) \rightarrow \mathbf{R}$ by

$$
f(\gamma)=-\frac{p^{*}(s)-p}{p(p-1)}|\gamma|^{2-p} \Gamma_{\mu}(\gamma)
$$


Then by (3.22) and (3.25), both $H_{1}$ and $H_{2}$ are solutions to the following initial value problem

$$
\left\{\begin{array}{l}
H^{\prime}(t)=f(H(t)) \quad \text { for }(t, H) \in I \times\left(\gamma_{1}, \gamma_{2}\right), \\
H(0)=\delta .
\end{array}\right.
$$

In equation (3.31), $I=\mathbf{R}$ in the case $0 \leq \mu<\bar{\mu}$, and $I=\mathbf{R} \backslash\left\{t_{-}, t_{-}^{\prime}\right\}$ in the case $-\infty<\mu<0$, where $t_{-}<0$ is the number defined as in Lemma 3.2 with respect to $z_{1}$ and $t_{-}^{\prime}<0$ the number with respect to $z_{2}$.

So we have two cases: Case 1: $0 \leq \mu<\bar{\mu}$; Case 2: $-\infty<\mu<0$.

In Case 1, we have $I=\mathbf{R}$ in equation (3.31). Note that in this case, $0 \leq \gamma_{1}<\delta<$ $\gamma_{2}$. Then $f \in C^{1}\left(\gamma_{1}, \gamma_{2}\right)$. Hence $f$ is locally Lipshitz in $\left(\gamma_{1}, \gamma_{2}\right)$. Then by Lemma B.1 (1), equation (3.31) admits at most one solution. Hence $H_{1} \equiv H_{2}$ on $\mathbf{R}$. It follows from equation (3.27) that $y_{1} \equiv y_{2}$ on $\mathbf{R}$. So the uniqueness in Case 1 is proved.

In case 2 , we have that $I=\mathbf{R} \backslash\left\{t_{-}, t_{-}^{\prime}\right\}$ in equation (3.31). Note that in this case $0 \in\left(\gamma_{1}, \gamma_{2}\right)$.

We divide the proof into three cases: Case 2.1: $p=2$; Case 2.2: $1<p<2$; Case 2.3: $2<p<N$.

In Case 2.1, $f(\gamma)=-\left(2^{*}(s)-2\right)\left(\gamma^{2}-(N-2) \gamma+\mu\right) / 2$. It is obvious that $f \in C^{1}\left(\gamma_{1}, \gamma_{2}\right)$. Hence $f$ is locally Lipshitz in $\left(\gamma_{1}, \gamma_{2}\right)$. So we can prove that $y_{1} \equiv y_{2}$ on $\mathbf{R}$ in the same way as that of Case 1. The uniqueness in Case 2.1 is proved.

In Case 2.2, $f$ is not Lipshitz in any neighborhood of $\gamma=0$. We can not use above argument. Let $y=y_{1}-y_{2}$ and $z=z_{1}-z_{2}$. Then $y$ satisfies equation

$$
\left\{\begin{array}{l}
\left(e^{-\delta t} y\right)^{\prime}=e^{-\delta t}\left(\left|z_{1}\right|^{\frac{1}{p-1}-1} z_{1}-\left|z_{2}\right|^{\frac{1}{p-1}-1} z_{2}\right) \text { in } \mathbf{R}, \\
y(0)=0
\end{array}\right.
$$

and $z$ satisfies equation

$$
\left\{\begin{array}{l}
\left(e^{\delta t} z\right)^{\prime}=e^{\delta t}\left(y_{2}^{p^{*}(s)-1}-y_{1}^{p^{*}(s)-1}+\mu y_{2}^{p-1}-\mu y_{1}^{p-1}\right) \text { in } \mathbf{R}, \\
z(0)=0 .
\end{array}\right.
$$

Fix a number $T, T>0$. Since $1<p<2$, the function $|t|^{\frac{1}{p-1}-1} t$ is continuously differentiable on $\mathbf{R}$. We have that

$$
\left.|| z_{1}\right|^{\frac{1}{p-1}-1} z_{1}-\left|z_{2}\right|^{\frac{1}{p-1}-1} z_{2}\left|\leq\left(\frac{1}{p-1} \sup _{\tau \in \mathbf{R}}|\tau|^{\frac{2-p}{p-1}}\right)\right| z_{1}-z_{2}\left|=: C_{3}\right| z \mid \quad \text { on }[-T, T] .
$$

Recall that $0<y_{i}(t)$ for all $i=1,2$. Hence $\inf _{[-T, T]} y_{1}>0$ and $\inf _{[-T, T]} y_{2}>0$. We have that

$$
\left|y_{2}^{p^{*}(s)-1}-y_{1}^{p^{*}(s)-1}\right|+\left|\mu y_{2}^{p-1}-\mu y_{1}^{p-1}\right| \leq C_{4}\left|y_{1}-y_{2}\right|=C_{4}|y| \quad \text { on }[-T, T],
$$

where $C_{4}>0$ is a constant depending on $N, p, \mu, s, \inf _{[-T, T]} y_{1}$ and $\inf _{[-T, T]} y_{2}$. Let $C_{T}=\max \left(C_{3}, C_{4}\right)$. Then by equation (3.33) we obtain that

$$
e^{\delta t}|z(t)|=\left|\int_{0}^{t}\left(e^{\delta \tau} z(\tau)\right)^{\prime} d \tau\right| \leq C_{T} \int_{0}^{t} e^{\delta \tau}|y(\tau)| d \tau \quad \text { for } 0<t<T .
$$

Write $Y(t)=e^{-\delta t} y(t)$ for $t \in \mathbf{R}$. By equation (3.32) and above estimate, we obtain that

$$
|Y(t)| \leq C_{T}^{2} t \int_{0}^{t}|Y(\tau)| d \tau \quad \text { for } 0<t<T
$$

Since $Y(0)=0$, it follows from the well known Gronwall's inequality that $Y \equiv 0$ in $[0, T]$. Hence $y \equiv 0$ on $[0, T]$. We can prove similarly that $y \equiv 0$ on $[-T, 0]$. 
Since $T>0$ is arbitrary, we obtain that $y \equiv 0$ on $\mathbf{R}$. So the uniqueness is proved in Case 2.2 .

It remains to consider Case 2.3. First we prove that $t_{-}=t_{-}^{\prime}$. With no loss of generality, we assume that $t_{-}^{\prime} \leq t_{-}<0$. Then both $z_{1}$ and $z_{2}$ do not change sign in the interval $\left(t_{-}, \infty\right)$. Precisely, both $z_{1}$ and $z_{2}$ are negative in $\left(t_{-}, \infty\right)$. Then the function $\left|z_{i}(t)\right|^{\frac{1}{p-1}-1} z_{i}(t)$ is continuously differentiable in $\left(t_{-}, \infty\right)$. We can apply the same argument as that of Case 2.2 to show that $y \equiv 0$ in $\left(t_{-}, \infty\right)$. Then it follows from $(3.29)$ that $z \equiv 0$ in $\left(t_{-}, \infty\right)$. In particular, we have that $z_{2}\left(t_{-}\right)=z_{1}\left(t_{-}\right)=0$. Hence we apply Lemma $3.2(3)$ to $z_{2}$ and obtain that $t_{-}^{\prime}=t_{-}$. Thus in case 2.3 we have that $I=\mathbf{R} \backslash\left\{t_{-}\right\}$.

We still need to show that $y_{1} \equiv y_{2}$ in $\left(-\infty, t_{-}\right)$. Consider the following initial value problem

$$
\left\{\begin{array}{l}
H^{\prime}(t)=f(H(t)) \quad \text { for }(t, H) \in\left(-\infty, t_{-}\right) \times\left(\gamma_{1}, \gamma_{2}\right) \\
H\left(t_{-}\right)=0
\end{array}\right.
$$

Then both $H_{1}$ and $H_{2}$ are nondecreasing solutions to equation $(3.35)$ in $\left(-\infty, t_{-}\right)$. Note that $\Gamma_{\mu}$ is strictly decreasing in $\left(\gamma_{1}, 0\right)$. Hence in Case $2.3, f$ is strictly increasing in $\left(\gamma_{1}, 0\right)$. Then by Lemma B.1, equation (3.35) admits at most one nondecreasing solution in $\left(-\infty, t_{-}\right)$. Hence $H_{1} \equiv H_{2}$ in $\left(-\infty, t_{-}\right)$. It follows from $(3.27)$ that $y_{1} \equiv y_{2}$ in $\left(-\infty, t_{1}\right)$. This completes the proof for Case 2.3 and so the proof of Theorem 1.4 is complete.

\section{Proof of Theorem 1.3}

In this section, we prove Theorem 1.3. Let $u \in W_{0}^{1, p}(B)$ be a positive radial solution to equation (1.1). Recall that we assume that $B$ is the unit ball in $\mathbf{R}^{N}$ centered at the origin. Then $u$ is a solution to the following ordinary differential equation

$$
\begin{cases}-\left(r^{N-1}\left|u^{\prime}(r)\right|^{p-2} u^{\prime}(r)\right)^{\prime}=\left(\frac{\mu}{r^{p}}+\frac{u(r)^{p^{*}(s)-p}}{r^{s}}+\lambda\right) u(r)^{p-1} r^{N-1}, & r \in(0,1) \\ u(r)>0, & r \in(0,1) \\ u(1)=0 & \end{cases}
$$

Since $u \in W_{0}^{1, p}(B)$, we have

$$
\int_{0}^{1}\left(|u(r)|^{p}+\left|u^{\prime}(r)\right|^{p}\right) r^{N-1} d r=\frac{1}{\omega_{N-1}} \int_{B}\left(|u|^{p}+|\nabla u|^{p}\right) d x<\infty
$$

where $\omega_{N-1}$ is the surface measure of the unit sphere in $\mathbf{R}^{N}$.

Before proving Theorem 1.3, we remark that in fact both $u$ and $r^{N-1}\left|u^{\prime}\right|^{p-2} u^{\prime}$ are continuously differentiable in $(0,1)$, and equation $(4.1)$ can be understood in the classical sense. Indeed, it is well known that every radially symmetric function in $W_{0}^{1, p}(B)$, after modifying on a set of measure zero, is a continuous function in $(0,1)$. Then by equation (4.1) we deduce that $r^{N-1}\left|u^{\prime}\right|^{p-2} u^{\prime} \in C^{1}(0,1)$. Thus equation (4.1) can be understood in the classical sense.

We prove Theorem 1.3 now. We only prove Theorem 1.3 in the case $0<\mu<\bar{\mu}$. We can prove Theorem 1.3 in the case $\mu \leq 0$ similarly. In the case when $0<\mu<\bar{\mu}$, the same estimates were obtained by the authors in [20] for positive radial weak 
solutions to the following equation

$$
-\Delta_{p} u-\frac{\mu}{|x|^{p}}|u|^{p-2} u=f(u)-m|u|^{p-2} u, \quad \text { in } \mathbf{R}^{N},
$$

where $f$ satisfies the growth condition $|f(t)| \leq C\left(|t|^{p-1}+|t|^{p^{*}-1}\right)$ for all $t \in \mathbf{R}$ by the assumptions therein. Theorem 1.3 can be proved by the same argument as that of [20, Theorem 1.1]. For the sake of completeness, we mimic a proof here.

Proof of Theorem 1.3. Let $u \in W_{0}^{1, p}(B)$ be a positive radial solution to equation (1.1) with $0<\mu<\bar{\mu}$ in the following. To start with we claim that

$$
u^{\prime}(r)<0 \text { for } r \text { sufficiently small. }
$$

Indeed, note that since $u \in W_{0}^{1, p}(B)$ is a radial function, we have by [25, Corollary II.1] that

It follows that

$$
u(r) r^{\frac{N-p}{p}}=o(1) \quad \text { as } r \rightarrow 0 .
$$

$$
u(r)^{p^{*}(s)-p} r^{p-s}=o(1) \quad \text { as } r \rightarrow 0 .
$$

Hence

$$
\left(\frac{\mu}{r^{p}}+\frac{u(r)^{p^{*}(s)-p}}{r^{s}}+\lambda\right) u(r)^{p-1} r^{N-1}>\frac{\mu}{2} u(r)^{p-1} r^{N-p-1}>0 \quad \text { for } r \text { small enough. }
$$

Therefore $\left(r^{N-1}\left|u^{\prime}\right|^{p-2} u^{\prime}\right)^{\prime}<0$ for $r$ small enough by equation (4.1). Hence $r^{N-1}\left|u^{\prime}\right|^{p-2} u^{\prime}$ is strictly decreasing for $r$ small enough. So we can assume that $\lim _{r \rightarrow 0} r^{N-1}\left|u^{\prime}\right|^{p-2} u^{\prime}=a$ for some $a \in(-\infty, \infty]$. We will prove that $a=0$. Suppose, on the contrary, that $a \neq 0$. Then there exist constants $C, r_{0}>0$ such that $\left|u^{\prime}(r)\right| \geq C r^{-\frac{N-1}{p-1}}$ for $0<r<r_{0}$. Then we have

$$
\int_{0}^{r_{0}}\left|u^{\prime}(r)\right|^{p} r^{N-1} d r \geq C \int_{0}^{r_{0}} r^{-\frac{N-1}{p-1}} d r=\infty .
$$

We reach a contradiction to (4.2). Hence $a=0$. Therefore $r^{N-1}\left|u^{\prime}\right|^{p-2} u^{\prime}<0$ for $r$ small enough. This proves (4.3).

Consider the function

$$
w(r)=-\frac{r^{p-1}\left|u^{\prime}(r)\right|^{p-2} u^{\prime}(r)}{u^{p-1}(r)} \quad \text { for } r>0 .
$$

Then $w \in C^{1}(0,1), w(r)>0$ for $r>0$ small enough by (4.3), and $w$ satisfies

$$
w^{\prime}(r)=\frac{1}{r}\left(\Gamma_{\mu}\left(w(r)^{\frac{1}{p-1}}\right)+u(r)^{p^{*}(s)-p} r^{p-s}+\lambda r^{p}\right) .
$$

Recall that $\Gamma_{\mu}$ is defined as in (1.8). To prove Theorem 1.3, it is enough to prove that

$$
w(r)=\gamma_{1}^{p-1}+o\left(r^{\delta}\right) \quad \text { as } r \rightarrow 0,
$$

for some $\delta \in(0,1)$. Here $\gamma_{1}$ is defined as in (1.9). In the case when $0<\mu<\bar{\mu}$, we note that $0<\gamma_{1}<(N-p) / p$.

First, we prove that $\lim _{r \rightarrow 0} w(r)$ exists and

$$
\lim _{r \rightarrow 0} w(r)=\gamma_{1}^{p-1}
$$

To prove that $\lim _{r \rightarrow 0} w(r)$ exists, we suppose, on the contrary, that

$$
\beta \equiv \limsup _{r \rightarrow 0} w(r)>\liminf _{r \rightarrow 0} w(r) \equiv \alpha .
$$


Then there exist two sequences of positive numbers $\left\{\xi_{i}\right\}$ and $\left\{\eta_{i}\right\}$ such that $\xi_{i} \rightarrow 0$ and $\eta_{i} \rightarrow 0$ and that $\eta_{i}>\xi_{i}>\eta_{i+1}$ for all $i=1,2, \cdots$. Moreover, the function $w$ has a local maximum at $\xi_{i}$ and a local minimum at $\eta_{i}$ for all $i=1,2, \cdots$, and

$$
\lim _{i \rightarrow \infty} w\left(\xi_{i}\right)=\beta, \quad \lim _{i \rightarrow \infty} w\left(\eta_{i}\right)=\alpha .
$$

Note that $w^{\prime}\left(\xi_{i}\right)=w^{\prime}\left(\eta_{i}\right)=0$. By equation (4.6), we have that

$$
\Gamma_{\mu}\left(w^{\frac{1}{p-1}}\left(\xi_{i}\right)\right)+\lambda \xi_{i}^{p}+u\left(\xi_{i}\right)^{p^{*}(s)-p} \xi_{i}^{p-s}=0,
$$

and that

$$
\Gamma_{\mu}\left(w^{\frac{1}{p-1}}\left(\eta_{i}\right)\right)+\lambda \eta_{i}^{p}+u\left(\eta_{i}\right)^{p^{*}(s)-p} \eta_{i}^{p-s}=0 .
$$

By (4.4) and the above two equalities, we have that

$$
\lim _{i \rightarrow \infty} \Gamma_{\mu}\left(w^{\frac{1}{p-1}}\left(\xi_{i}\right)\right)=\lim _{i \rightarrow \infty} \Gamma_{\mu}\left(w^{\frac{1}{p-1}}\left(\eta_{i}\right)\right)=0 .
$$

Since $\Gamma_{\mu}(s) \rightarrow \infty$ as $|s| \rightarrow \infty,\left\{w\left(\xi_{i}\right)\right\}$ and $\left\{w\left(\eta_{i}\right)\right\}$ are bounded. So $\alpha, \beta$ are finite and

$$
\Gamma_{\mu}\left(\beta^{\frac{1}{p-1}}\right)=\Gamma_{\mu}\left(\alpha^{\frac{1}{p-1}}\right)=0 .
$$

Recall that $\Gamma_{\mu}(\gamma)=0$ if and only if $\gamma=\gamma_{1}$ or $\gamma=\gamma_{2}$. Recall also that in the case when $0<\mu<\bar{\mu}$, we have $0<\gamma_{1}<(N-p) / p<\gamma_{2}<(N-p) /(p-1)$. Hence

$$
\beta=\gamma_{2}^{p-1} \text { and } \quad \alpha=\gamma_{1}^{p-1} \text {. }
$$

That is,

$$
\lim _{i \rightarrow \infty} w\left(\xi_{i}\right)=\gamma_{2}^{p-1} \text { and } \quad \lim _{i \rightarrow \infty} w\left(\eta_{i}\right)=\gamma_{1}^{p-1} .
$$

Note that $\gamma_{1}<(N-p) / p<\gamma_{2}$. So there exists $\zeta_{i} \in\left(\eta_{i+1}, \xi_{i}\right)$ such that

$$
w\left(\eta_{i+1}\right)<w\left(\zeta_{i}\right)=\left(\frac{N-p}{p}\right)^{p-1}<w\left(\xi_{i}\right)
$$

for $i$ large enough. Then by (4.4) and equation (4.6), we obtain that

$$
\zeta_{i} w^{\prime}\left(\zeta_{i}\right)=\Gamma_{\mu}\left(\frac{N-p}{p}\right)+\lambda \zeta_{i}^{p}+u\left(\zeta_{i}\right)^{p^{*}(s)-p} \zeta_{i}^{p-s}=-(\bar{\mu}-\mu)+o(1)<0
$$

for $i$ large enough. Here we used the fact that

$$
\Gamma_{\mu}\left(\frac{N-p}{p}\right)=-(\bar{\mu}-\mu) .
$$

Hence $w^{\prime}\left(\zeta_{i}\right)<0$ for $i$ large enough. Therefore $w$ is strictly decreasing in a neighborhood of $\zeta_{i}$. Since $\zeta_{i}<\xi_{i}$ and $w\left(\zeta_{i}\right)<w\left(\xi_{i}\right)$, there exists $\zeta_{i}<\zeta_{i}^{\prime}<\xi_{i}$ such that $w(r) \leq w\left(\zeta_{i}\right)$ for $\zeta_{i}<r<\zeta_{i}^{\prime}$ and $w\left(\zeta_{i}^{\prime}\right)=w\left(\zeta_{i}\right)$. Thus $w^{\prime}\left(\zeta_{i}^{\prime}\right) \geq 0$. However, by equation (4.6), we have that $w^{\prime}\left(\zeta_{i}^{\prime}\right)<0$. We reach a contradiction. Therefore $\lim _{r \rightarrow 0} w(r)$ exists.

Set $k^{p-1}=\lim _{r \rightarrow 0} w(r)$. We will prove that $k=\gamma_{1}$. We claim that $k \leq(N-p) / p$. Otherwise, choose $\epsilon>0$ such that $k-\epsilon>(N-p) / p$. Then for $r$ small enough we have $w(r)>(k-\epsilon)^{p-1}$, that is, $-r u^{\prime}(r) / u(r)>k-\epsilon$ for $r$ small enough. This implies that $u(r) \geq C r^{\epsilon-k}$ for $r$ small enough, which implies $u \notin L^{p^{*}}(B)$. We reach a contradiction. Thus $k \leq(N-p) / p$.

By (4.4) and equation (4.6), we have that

$$
\lim _{r \rightarrow 0} r w^{\prime}(r)=\Gamma_{\mu}(k) .
$$


We claim that $\Gamma_{\mu}(k)=0$. Otherwise, suppose that $\Gamma_{\mu}(k) \neq 0$. Note that for any $0<t<s_{0}$, we have

$$
w\left(s_{0}\right)=w(t)+\int_{t}^{s_{0}} w^{\prime}(t) d t
$$

Then $\Gamma_{\mu}(k) \neq 0$ implies that $\lim _{t \rightarrow 0}\left|\int_{t}^{s_{0}} w^{\prime}(t) d t\right|=\infty$ if $s_{0}$ is small enough. This contradicts to (4.8). Hence $\Gamma_{\mu}(k)=0$. Recall that $\Gamma_{\mu}(\gamma)=0$ if and only if $\gamma=\gamma_{1}$ or $\gamma=\gamma_{2}$. Thus we have either $k=\gamma_{1}$ or $k=\gamma_{2}$. Then we deduce that $k=\gamma_{1}$ since $k \leq(N-p) / p<\gamma_{2}$. This proves (4.8).

As a result, (4.8) implies that for any $\epsilon>0$ sufficiently small there exist $C, c>0$ such that

$$
c r^{-\gamma_{1}+\epsilon} \leq u(r) \leq C r^{-\gamma_{1}-\epsilon}
$$

for $r>0$ small enough. Choose $\epsilon=\epsilon_{0}>0$ such that $p-s-\left(p^{*}(s)-p\right)\left(\gamma_{1}+\epsilon_{0}\right)>0$. This is possible since $\gamma_{1}<(N-p) / p$. Applying (4.4), we obtain that

$$
u(r)^{p^{*}(s)-p} r^{p-s} \leq C r^{p-s-\left(p^{*}(s)-p\right)\left(\gamma_{1}+\epsilon_{0}\right)} \equiv C r^{\delta_{0}}
$$

for $r>0$ small enough. Here $\delta_{0}=p-s-\left(p^{*}(s)-p\right)\left(\gamma_{1}+\epsilon_{0}\right)>0$.

Now we prove (4.7). Let $w_{1}(r)=w(r)-\gamma_{1}^{p-1}$. Then $w_{1}(r) \rightarrow 0$ as $r \rightarrow 0$. We prove that $w_{1}(r)=o\left(r^{\delta}\right)$ as $r \rightarrow 0$ for some $\delta>0$. By equation (4.6) and the definition of $\Gamma_{\mu}$ (see (1.8)), we have

$$
\begin{aligned}
w_{1}^{\prime}(r) & =w^{\prime}(r)=\frac{1}{r} \Gamma_{\mu}\left(w^{\frac{1}{p-1}}(r)\right)+\frac{1}{r}\left(u(r)^{p^{*}(s)-p} r^{p-s}+\lambda r^{p}\right) \\
& =\frac{1}{r}\left((p-1) w^{\frac{p}{p-1}}(r)-(N-p) w(r)+\mu\right)+\frac{1}{r}\left(u(r)^{p^{*}(s)-p} r^{p-s}+\lambda r^{p}\right) \\
& =\frac{A(r)}{r} w_{1}(r)+B(r),
\end{aligned}
$$

for $r$ small enough, where $A(r) \rightarrow p \gamma_{1}-(N-p)<0$ as $r \rightarrow 0$ and

$$
B(r)=\frac{1}{r}\left(u(r)^{p^{*}(s)-p} r^{p-s}+\lambda r^{p}\right)=O\left(r^{\delta_{0}-1}\right) \quad \text { as } r \rightarrow 0,
$$

by (4.9). Here $\delta_{0}>0$ is defined as in (4.9).

Fix $r_{0}>0$ small and define $h(r)=\int_{r}^{r_{0}} A(\tau) \tau^{-1} d \tau$ for $0<r<r_{0}$. Since $w_{1}$ is a solution to equation (4.10), it has the following form

$$
w_{1}(r)=\int_{0}^{r} e^{h(t)-h(r)} B(t) d t
$$

Since $h(t)-h(r)=\int_{t}^{r} A(\tau) \tau^{-1} d \tau<0$ for $0<t<r$, we obtain that $e^{h(t)-h(r)} \leq 1$ for $0<t<r$. Hence by (4.11), we have for $r$ small enough that

$$
\left|w_{1}(r)\right| \leq \int_{0}^{r}|B(t)| d t \leq C r^{\delta_{0}}
$$

Here $\delta_{0}>0$ is as in (4.9). This proves (4.7).

Recall that $w$ is defined as (4.5). The conclusion of Theorem 1.3 follows easily from estimate (4.7). The proof of Theorem 1.3 in the case $0<\mu<\bar{\mu}$ is complete. 


\section{Appendix A. A comparison result}

Let $\Omega$ be a bounded domain in $\mathbf{R}^{N}$ containing the origin. Define the operator $-L_{p}$ by

$$
-L_{p} u=-\Delta_{p} u-\frac{\mu}{|x|^{p}}|u|^{p-2} u, \quad u \in W_{0}^{1, p}(\Omega) .
$$

We have the following result.

Lemma A.1. Let $\rho_{1}, \rho_{2}$ be two nonnegative functions in $L^{\frac{N}{p}}(\Omega)$. Denote by $\lambda_{1}\left(\rho_{i}\right)$ the first eigenvalue of the operator $-L_{p}$ with respect to weight $\rho_{i}, i=1,2$, that is,

$$
\lambda_{1}\left(\rho_{i}\right)=\inf \left\{\frac{Q(\varphi)}{\int_{\Omega} \rho_{i}|\varphi|^{p} d x}: \varphi \in W_{0}^{1, p}(\Omega), \varphi \neq 0\right\}
$$

for $i=1,2$, where

$$
Q(\varphi)=\int_{\Omega}\left(|\nabla \varphi|^{p}-\frac{\mu}{|x|^{p}}|\varphi|^{p}\right) d x
$$

If $\rho_{1} \geq \rho_{2}$, then either $\lambda_{1}\left(\rho_{1}\right)<\lambda_{1}\left(\rho_{2}\right)$ or $\lambda_{1}\left(\rho_{1}\right)=\lambda_{1}\left(\rho_{2}\right)$ and $e_{i}=0(i=1,2)$ on $\left\{x \in \Omega: \rho_{1}(x) \neq \rho_{2}(x)\right\}$, where $e_{1}, e_{2}$ are the first eigenfunctions corresponding to the first eigenvalues $\lambda_{1}\left(\rho_{1}\right)$ and $\lambda_{1}\left(\rho_{2}\right)$ respectively.

Proof. In the case when $\mu=0$, Lemma A.1 was proved by Adimurthi and Yadava [1, Lemma 4.1]. Their argument can be easily applied to prove Lemma A.1. For completeness, we give a proof here.

It is direct to verify that $\lambda_{1}\left(\rho_{1}\right) \leq \lambda_{1}\left(\rho_{2}\right)$ by definition. Suppose that $\lambda_{1}\left(\rho_{1}\right)=$ $\lambda_{1}\left(\rho_{2}\right)$ and $e_{1}, e_{2}$ are the first eigenfunctions corresponding to the first eigenvalues $\lambda_{1}\left(\rho_{1}\right)$ and $\lambda_{1}\left(\rho_{2}\right)$ respectively. Then $e_{i}, i=1,2$, are nonpositive or nonnegative functions in $\Omega$. We assume that $e_{i} \geq 0$ for both $i=1,2$. Then

$$
\lambda_{1}\left(\rho_{i}\right)=\frac{Q\left(e_{i}\right)}{\int_{\Omega} \rho_{i}\left|e_{i}\right|^{p} d x}, \quad i=1,2 .
$$

Therefore,

$$
\frac{Q\left(e_{2}\right)}{\int_{\Omega} \rho_{1}\left|e_{2}\right|^{p} d x} \geq \frac{Q\left(e_{1}\right)}{\int_{\Omega} \rho_{1}\left|e_{1}\right|^{p} d x}=\lambda_{1}\left(\rho_{1}\right)=\lambda_{1}\left(\rho_{2}\right)=\frac{Q\left(e_{2}\right)}{\int_{\Omega} \rho_{2}\left|e_{2}\right|^{p} d x} .
$$

Since $\rho_{1} \geq \rho_{2}$, we obtain that $\int_{\Omega} \rho_{1}\left|e_{1}\right|^{p} d x=\int_{\Omega} \rho_{2}\left|e_{1}\right|^{p} d x$. That is,

$$
\int_{\Omega}\left(\rho_{1}-\rho_{2}\right)\left|e_{1}\right|^{p} d x=0
$$

Since $e_{1}$ is nonnegative in $\Omega$, we have that $e_{1}=0$ on $\left\{x \in \Omega: \rho_{1}(x) \neq \rho_{2}(x)\right\}$.

Hence

$$
\lambda_{1}\left(\rho_{2}\right)=\lambda_{1}\left(\rho_{1}\right)=\frac{Q\left(e_{1}\right)}{\int_{\Omega} \rho_{1}\left|e_{1}\right|^{p} d x}=\frac{Q\left(e_{1}\right)}{\int_{\Omega} \rho_{2}\left|e_{1}\right|^{p} d x},
$$

which implies that $e_{1}$ is also an eigenfunction of $\lambda_{1}\left(\rho_{2}\right)$. Thus $e_{1}=k e_{2}$ for some $k \neq 0$ (see [35]). Thus $e_{2}=0$ on $\left\{x \in \Omega: \rho_{1}(x) \neq \rho_{2}(x)\right\}$. This finishes the proof of Lemma A.1. 


\section{Appendix B. A uniqueness result on ordinary differential equations}

The following result can be found in standard textbooks on ordinary differential equations.

Lemma B.1. Let $(a, b) \subset \mathbf{R}$ and $(c, d) \subset \mathbf{R}$ be two intervals. Assume that $f: \mathbf{R} \rightarrow \mathbf{R}$ be a continuous function. Consider the initial value problem

$$
\left\{\begin{array}{l}
y^{\prime}(t)=f(y(t)), \quad(t, y) \in(a, b) \times(c, d), \\
y\left(t_{0}\right)=y_{0},
\end{array}\right.
$$

for some $\left(t_{0}, y_{0}\right) \in(a, b) \times(c, d)$. Then we have

(1) if $f$ is locally Lipshcitz continuous in $(c, d)$, equation (B.1) admits at most one solution on $(a, b)$;

(2) if $f$ is nonincreasing in $\left(y_{0}, d\right)$, then equation (B.1) admits at most one nondecreasing solution on $\left(t_{0}, b\right)$;

(3) if $f$ is nondecreasing in $\left(c, y_{0}\right)$, then equation (B.1) admits at most one nondecreasing solution on $\left(a, t_{0}\right)$.

Proof. (1) can be proved in a standard way. We omit the details. We only prove conclusion (2). We can prove (3) similarly.

Suppose that $f$ is nonincreasing in $\left(y_{0}, d\right)$ and $y_{1}, y_{2}$ are two distinct nondecreasing solutions of equation (B.1) on $\left(t_{0}, b\right)$. With no loss of generality, we assume that $y_{1}\left(t_{1}\right)>y_{2}\left(t_{1}\right)$ for some $t_{1} \in\left(t_{0}, b\right)$. Let

$$
t_{2}=\inf \left\{t \in\left[t_{0}, t_{1}\right): y_{1}(s)>y_{2}(s) \text { for } s \in\left(t, t_{1}\right)\right\} \text {. }
$$

Then $t_{1}>t_{2} \geq t_{0}, y_{1}\left(t_{2}\right)=y_{2}\left(t_{2}\right)$, and $y_{1}(t)>y_{2}(t)$ for $t \in\left(t_{2}, t_{1}\right]$. Hence

$$
y_{1}^{\prime}(t)-y_{2}^{\prime}(t)=f\left(y_{1}(t)\right)-f\left(y_{2}(t)\right) \leq 0 \quad \text { for } t \in\left(t_{2}, t_{1}\right)
$$

since $f$ is nonincreasing in $\left(y_{0}, d\right)$. Thus $y_{1}-y_{2}$ is nonincreasing on $\left[t_{2}, t_{1}\right]$. In particular, we have that $y_{1}\left(t_{1}\right)-y_{2}\left(t_{1}\right) \leq y_{1}\left(t_{2}\right)-y_{2}\left(t_{2}\right)=0$. We reach a contradiction. This proves (2).

Acknowledgement. The authors would like to thank the referees for valuable comments. The second named author is financially supported by the Academy of Finland, project 259224.

\section{References}

[1] Adimurthi, and S.L. Yadava: An elementary proof of the uniqueness of positive radial solutions of a quasilinear Dirichlet problem. - Arch. Ration. Mech. Anal. 127:3, 1994, 219-229.

[2] Aubin, T.: Problèmes isopérimétriques de Sobolev. - J. Diff. Geom. 11:4, 1976, 573-598.

[3] Abdellaoui, B., V. Felli, and I. Peral: Existence and nonexistence results for quasilinear elliptic equations involving the $p$-Laplacian. - Boll. Unione Mat. Ital. Sez. B Artic. Ric. Mat. (8) $9: 2,2006,445-484$.

[4] Brézis, H., and L. Nirenberg: Positive solutions of nonlinear elliptic equations involving critical Sobolev exponents. - Comm. Pure Appl. Math. 36:4, 1983, 437-477.

[5] Caffarelli, L. A., B. Gidas, and J. Spruck: Asymptotic symmetry and local behavior of semilinear elliptic equations with critical Sobolev growth. - Comm. Pure Appl. Math. 42:3, 1989, 271-297.

[6] Caffarelli, L. A., R. Kohn, and L. Nirenberg: First order interpolation inequalities with weights. - Compos. Math. 53, 1984, 259-275.

[7] CAO, D., and P. HAN: Solutions for semilinear elliptic equations with critical exponents and Hardy potential. - J. Differential Equations 205:2, 2004, 521-537. 
[8] CaO, D., S. Peng, and S. YAn: Infinitely many solutions for $p$-Laplacian equation involving critical Sobolev growth. - J. Funct. Anal. 262:6, 2012, 2861-2902.

[9] CAO, D., and S. YAN: Infinitely many solutions for an elliptic problem involving critical Sobolev growth and Hardy potential. - Calc. Var. Partial Differential Equations 38:3-4, 2010, 471-501.

[10] Catrina, F., and Z. Q. WANG: On the Caffarelli-Kohn-Nirenberg inequalities: sharp constants, existence (and nonexistence), and symmetry of extremal functions. - Comm. Pure Appl. Math. 54:2, 2001, 229-258.

[11] Chou, K. S., and C. W. Chu: On the best constant for a weighted Sobolev-Hardy inequality. - J. London Math. Soc. (2) 48:1, 1993, 137-151.

[12] Franchi, B., E. Lanconelli, and J. Serrin: Existence and uniqueness of non negative solutions of quasilinear equations in $\mathbf{R}^{n}$. - Adv. Math. 118:2, 1996, 177-243.

[13] García Azorero, J. P., and I. Peral Alonso: Hardy inequalities and some critical elliptic and parabolic problems. - J. Differential Equations 144:2, 1998, 441-476.

[14] Gidas, B., W. M. Ni, and L. Nirenberg: Symmetry and related properties via the maximum principle. - Comm. Math. Phys. 68:3, 1979, 209-243.

[15] Guedda, M., and L. VÉron: Local and global properties of solutions of quasilinear elliptic equations. - J. Differential Equations 76:1, 1988, 159-189.

[16] Guedda, M., and L. VÉron: Quasilinear elliptic equations involving critical Sobolev exponents. - Nonlinear Anal. 13, 1989, 879-902.

[17] Ghoussoub, N., and C. YuAn: Multiple solutions for quasilinear PDEs involving critical Sobolev and Hardy exponents. - Trans. Amer. Math. Soc. 352, 2000, 5703-5743.

[18] Han, P.: Quasilinear elliptic problems with critical exponents and Hardy terms. - Nonlinear Anal. 61:5, 2005, 735-758.

[19] Hardy, G., J. E. Littrewood, and G. Polya: Inequalities. Second edition. - Cambridge, at the University Press, 1952.

[20] He, C.-J., and C.-L. XIANG: A note on asymptotic behaviors of solutions to quasilinear elliptic equations with Hardy potential. - Preprint, arXiv:1502.03969.

[21] Jannelli, E.: The role played by space dimension in elliptic critical problems. - J. Differential Equations 156:2, 1999, 407-426.

[22] Knate, M. C., and L. A. Peletier: Quasilinear elliptic equations with nearly critical growth. - Comm. Partial Differential Equations 14, 1989, 1351-1383.

[23] Kwong, M. K.: Uniqueness of positive solutions of $\Delta u-u+u^{p}=0$ in $\mathbf{R}^{n}$. - Arch. Ration. Mech. Anal. 105:3, 1989, 243-266.

[24] Lieb, E. H.: Sharp constants in the Hardy-Littlewood-Sobolev and related inequalities. - Ann. of Math. (2) 118:2, 1983, 349-374.

[25] Lions, P. L.: Symétrie et compacité dans les espaces de Sobolev. - J. Funct. Anal. 49:3, 1982, $315-334$.

[26] Ni, W. M., and J. SerRIN: Non-existence theorems for quasilinear partial differential equations. - Suppplemento di Rend. Circ. Mat. Palermo 8, 1985, 171-185.

[27] NI, W. M., and J. SERRIN: Existence and non-existence theorems for ground states of quasilinear partial differential equations. The anomalous case. - Rome Acc. Naz. dei Lincei, Atti dei Convegni 77, 1986, 231-257.

[28] PohožAev, S.: Eigenfunction of the equation. - Soviet Math. Dokl. 6, 1965, 1408-1411.

[29] Pucci, P., and J. Serrin: A general variational identity. - Indiana Univ. Math. J. 35, 1986, 681-703.

[30] Ramaswamy, M., and S. Santra: Uniqueness and profile of positive solutions of a critical exponent problem with Hardy potential. - J. Differential Equations 254:11, 2013, 4347-4372. 
[31] SRIKanth, P. N.: Uniqueness of solutions of nonlinear Dirichlet problems. - Differential Integral Equations 6:3, 1993, 663-670.

[32] Talenti, G.: Best constant in Sobolev inequality. - Ann. Mat. Pura Appl. (4) 110, 1976, $353-372$.

[33] Terracini, S.: On positive entire solutions to a class of equations with a singular coefficient and critical exponent. - Adv. Differential Equations 1:2, 1996, 241-264.

[34] Zhang, L. Q.: Uniqueness of positive solutions of $\Delta u+u+u^{p}=0$ in a ball. - Comm. Partial Differential Equations 17:7-8, 1992, 1141-1164.

[35] Zhang, G., X. WANG, and S. Liu: On a class of singular elliptic problems with perturbed Hardy-Sobolev operator. - Calc. Var. Partial Differential Equations 46, 2013, 97-111.

Received 23 March 2015 • Accepted 25 June 2015 